\title{
Competing responses and the processing of irrelevant information*
}

\author{
MILTON H. HODGE \\ University of Georgia, Athens, Georgia 30601
}

\begin{abstract}
A number of studies have shown that performance of identification and discrimination tasks is detrimentally affected by irrelevant information, yet other studies have failed to find such decrements. It is suggested that these contradictory findings depend on whether S must make difficult discriminations among the relevant stimuli, the irrelevant stimuli, or between the relevant and irrelevant stimuli. The role of irrelevant information in these tasks is to enhance or amplify the competing responses engendered by the difficult discriminations. Irrelevant information enhances competing responses by increasing the information processing requirements of a task. The results of various studies of irrelevant information seem to be in good agreement with the assertions.
\end{abstract}

In recent years, a number of investigations have sought to assess the effects of irrelevant information on the performance of complex identification and discrimination tasks. Some of these studies (e.g., Egeth, 1966; Hodge, 1959; Hodge \& Reid, 1971; Montague, 1965; Morgan \& Alluisi, 1967; Well, 1971) have found that irrelevant information degrades performance, while others (e.g., Archer, 1954; Fitts \& Biederman, 1965; Imai \& Garner, 1965; Morin, Forrin, \& Archer, 1961) have not. To account for the detrimental effects of irrelevant information, Hodge (1959) suggested that the occurrence of competing responses was a likely explanation. The results obtained with a complex visual task seemed to support this view. Montague (1965) subsequently demonstrated that the same explanation appeared to account for similar results in an auditory task. In a review of filtering in speeded classification tasks, Egeth (1967) labeled the explanation the competing-response (CR) hypothesis, and noted its applicability to several other studies, especially the Stroop (1935) color-naming task. However, Egeth and Bevan (1972) and Well (1971) have recently argued that the CR hypothesis is not unambiguously applicable to the results obtained by Hodge and Montague. They claim that Hodge and Montague confounded irrelevant information with task complexity. Furthermore, there also seems to be evidence that irrelevant information can degrade performance in the absence of competing responses (Morgan \& Alluisi, 1967; Well, 1971), a result in sharp contrast with those obtained by Archer (1954), Fitts and Biederman (1965), Imai and Garner (1965), and Morin et al (1961).

In view of the questions concerning the $C R$ hypothesis and given the results of Morgan and Alluisi (1967) and Well (1971), it seems appropriate to reanalyze the hypothesis and its role in the processing of

\footnotetext{
*The author wishes to thank Clyde E. Noble and Howard Egeth for their critical reading of the manuscript.
}

irrelevant information. The sequel will suggest that the CR hypothesis must be expanded somewhat and paired with the notion of information processing load in order to account for the effects of irrelevant information.

\section{NATURE OF IRRELEVANT INFORMATION}

By definition, irrelevant information consists of stimuli and/or stimulus dimensions that are not necessary to the performance of some task. For example, if $S$ is to classify a set of stimulus objects on the basis of shape, the presence of different colors among the stimuli would constitute irrelevant information. Logically, there are three possible effects of irrelevant information: it could facilitate performance of a task, it could degrade performance, or it could have no effect. Although all three effects are conceivable, most investigators have found that irrelevant information either produces or does not produce decrements in performance. In identification and discrimination experiments, irrelevant information has been manipulated in two major ways. On the one hand, it has been introduced in the form of extra stimuli which ostensibly are not related to the correct response. In this form, irrelevant information is analogous to noise and has been designated as never-relevant irrelevant information by Hodge (1959). Presumably, in sufficient amounts, such irrelevant information will produce performance decrements because it places a demand on S's information processing capabilities (Broadbent, 1958), whether in terms of gating or filtering requirements (Posner, 1964) or in terms of central processing. Parenthetically, Posner and Boies (1971) have recently suggested that gating operations may not require the use of central processing capacity. In the extreme case, the noise may be so intense to preclude even simple detection of the relevant stimuli, i.e., even gating mechanisms may fail. Such situations are occasionally encountered in ordinary radio and TV reception. In the less extreme case found in most 
laboratory studies, the presence of small to moderate amounts of never-relevant irrelevant information seems to have little effect on task performance. Archer (1954), for example, found that a complex visual task was not affected by 1.2, or 3 bits of such irrelevant information. Similar results have been obtained by Fitts and Biederman (1965), Imai and Garner (1965), and Morin et al (1961).

In other experiments, the same stimuli have served as both relevant and irrelevant information, leading Hodge (1959) to characterize these stimuli as sometimes relevant and sometimes irrelevant. This characterization of the stimuli is a consequence of certain contingent relationships among the total set of task stimuli. For example, in visual stimulus patterns, one or more of the dimensions may provide relevant or irrelevant information, depending on the specific value of the dimension. A color dimension might provide relevant information when its value is red, but irrelevant information when blue. In contrast to the results obtained with never-relevant irrelevant information, increasing the amount of sometimes-relevant irrelevant information produces systematic decrements in task performance (Hodge, 1959; Hodge \& Reid, 1971; Montague, 1965).

Implicit in both types of irrelevant information experiments is the notion that increasing the amount of irrelevant information serves to increase overall task complexity. Notwithstanding the criticism of Egeth and Bevan (1972) and Well (1971) that Hodge (1959) and Montague (1965) confounded irrelevant information with task complexity, it is difficult to imagine a situation in which the amount of relevant or irrelevant information can be varied independently of task complexity. In fact, any manipulation which produces a greater number of stimulus objects or dimensions seems to be a commonly accepted way of varying stimulus complexity (Goldstein, 1966). Comparable operations define response complexity. Changes in either stimulus or response complexity underlie the concept of task complexity (Noble, 1957). Archer (1954) apparently had these definitions in mind when he stated that. "The amounts of relevant and irrelevant information in a stimulus may vary independently of each other; task complexity is a function of either or both types of information [p. 313]."

Consider the Stroop (1935) color-naming task which Egeth (1967) and Egeth and Bevan (1972) view as a nearly ideal vehicle for testing the CR hypothesis. In this task, $\mathrm{S}$ is required to name the ink color of a series of printed words, but at the same time to ignore the fact that each word is the name of a color different from the ink color. For example, given the word blue printed in red ink. S should respond with red. Performance of an experimental group on this task was poorer than that of a control group asked to identify colored rectangles. In terms of the previous analysis. the experimental group had to deal with sometimes-relevant irrelevant information (the color names), while the control group had to filter out the never-relevant information provided by the rectangular shapes. Although it might be argued that the two tasks are equal in complexity (both have two dimensions pertinent to the task), the experimental task seems more complex because both dimensions were varied over a number of values. In contrast, only color was permitted to change in the control task. Thus. it seems reasonable to conclude, as Archer (1954) and implicitly as Hodge (1959). Hodge and Reid (1971), and Montague (1965) did, that the introduction of varying amounts of irrelevant information produces different degrees of task complexity. The two variables seem to be defined by similar, highly correlated events. On the other hand, it should not be forgotten that task complexity can also be varied by changing the amount of relevant information. Finally, it needs to be made explicit that greater information processing capacity is presumably necessary for complex tasks than for simple tasks. As more information, relevant or irrelevant. must be processed. more processing capacity is required.

Another variable which influences the processing of irrelevant information is the similarity among the task stimuli. Variations in stimulus similarity can occur among the relevant stimuli, among the irrelevant stimuli. and between the relevant and irrelevant stimuli. If such variations are actually present, $S$ must make discriminations whose ease or relative difficulty is a function of the number of stimuli in each of the three stimulus classes and the degree of similarity. Low similarity makes the discriminations easy, but high similarity tends to produce relatively difficult discriminations (Hodge, 1959; Hodge \& Reid, 1971: Montague, 1965). The similarity variable seems to be particularly potent if $S$ must contend with a large number of discriminations, especially if he is under pressure to respond quickly. Further discussion of the relationship between similarity and processing load is postponed to the next section.

\section{CR HYPOTHESIS}

The notion of competing responses or interference is directly derivable from S-R interpretations of retention and transfer (Ellis, 1965; McGeoch \& Irion, 1952; Postman, 1961) and is generally assumed to underlie the decremental phenomena of negative transfer and forgetting. Among the factors known to influence these phenomena, perhaps the most potent is similarity (stimulus and response). An examination of the Osgood (1949) transfer and retroaction surface shows that maximum interference is likely to occur when antagonistic responses (minimum response similarity) are made to the same (identical) stimuli (maximum stimulus similarity). Interference tends to decline with increases in response similarity and with decreases in stimulus similarity. Although the predictive value of the Osgood surface has been questioned (see Ellis. 1965). especially 
with respect to response similarity. it is nonetheless a useful first approximation to similarity effects. It should also be noted that the occurrence of competing responses is presumably enhanced if the stimuli and responses occur in reasonably close temporal contiguity. The $S$ is not likely to make erroneous responses if he has sufficient time to make careful discriminations among the stimuli and responses.

Consider now the applicability of the CR hypothesis to the detrimental effects of irrelevant information, keeping in mind that competing responses are presumably enhanced whenever $S$ is required to make different responses to the same stimuli and that performance should improve with a decrease in stimulus similarity. With less similar stimuli. S should find it easier to discriminate among the task stimuli and thus easier to associate each stimulus with a unique and independent response, thereby reducing interference. Applied to the irrelevant information studies, this analysis suggests that performance should decline with: (a) increasing discrimination difficulty among the relevant or the irrelevant stimuli and (b) increasing discrimination difficulty between the relevant and irrelevant stimuli. Although discrimination difficulty is mainly a function of stimulus similarity, the discriminative capacity of $S$ is presumably limited and thus sensitive to the total processing load imposed by a particular task. Hence, increasing the processing load by the introduction of more stimulus objects and/or dimensions, whether relevant or irrelevant, should also lead to performance decrements. More specifically, the major consequence of increasing the processing load appears to be amplification of the detrimental effects engendered by the difficult discriminations.

The major points of the preceding analysis are that competing responses occur primarily as the result of difficult stimulus discriminations and that the relevancy-irrelevancy of particular stimuli is of secondary importance. Whether stimuli are relevant or irrelevant in an experimental task is usually based on an arbitrary decision by the investigator. Irrelevant stimuli can degrade task performance in at least two ways. On the one hand. these stimuli are of ten highly similar to the relevant information and thus require a difficult discrimination, which in turn contributes to the arousal of competing responses. This is the situation which employs sometimes-relevant irrelevant information. Irrelevant information may also influence performance by amplifying the competition produced by difficult discriminations among the relevant stimuli. This possibility seems particularly likely in tasks which involve never-relevant irrelevant information. Just how the amplification takes place is not clear, but, as was suggested earlier. the irrelevant information may simply increase the overall processing load. The suggestion is reasonable if it is assumed that some portion of central processing capacity is devoted to gating or filtering the irrelevant stimuli. The limitation suggested by Posner and Boies (1971), that gating operations do not involve central processing capacity. may not be applicable in situations with a large number of complex irrelevant stimuli. Gating of the simple stimuli used by Posner and Boies may well not have required central processing capacity. However. to the extent that central processing capacity is used in gating never-relevant irrelevant stimuli. less capacity is available for processing the relevant stimuli. particularly if the relevant stimuli require difficult discriminations. Such conditions should enhance the likelihood of interference effects.

\section{EVIDENCE}

How well do these assertions agree with the experimental evidence? Examine first the early studies which showed that task performance is not affected by never-relevant irrelevant information (Archer, 1954; Fitts \& Biederman, 1965; Imai \& Garner, 1965; Morin et al, 1961). In none of these studies is there much evidence for response competition. The Ss were not required to make difficult discriminations among the relevant stimuli or between the relevant and irrelevant stimuli. Nor were Ss required to perform difficult gating operations on complex irrelevant stimuli. As Hodge and Reid (1971) point out, Ss in the Imai and Garner experiment did have to distinguish whether a given stimulus dimension was relevant or irrelevant in a block of trials, but never from trial to trial.

Consider next the investigations explicitly designed to test the CR hypothesis (Hodge, 1959: Hodge \& Reid, 1971; Montague, 1965). Among the variables manipulated in these experiments were the amount of sometimes-relevant irrelevant information, difficulty of discrimination between the relevant and irrelevant stimuli (Hodge \& Reid, 1971), and difficulty of discrimination among some of the relevant stimuli (form dimension in Hodge, 1959; pitch dimension in Montague, 1965). In each case. the irrelevant information produced significant declines in task performance. The difficulty of discrimination manipulations also led to performance decrements and thus support the assertion that discrimination difficulty (high stimulus similarity) plays a major role in the arousal of competing responses. Furthermore, this result was obtained with both types of discrimination difficulty (among the relevant stimuli and between the relevant and irrelevant stimuli). More importantly, each of the studies also showed a significant interaction between discrimination difficulty and amount of irrelevant information, supporting the argument that increasing amounts of irrelevant information serve to amplify or enhance the competition engendered by the similarity variable.

In the Morgan and Alluisi (1967) experiment, performance declined systematically with increasing similarity among the relevant stimuli. but the decline became progressively worse with increasing amounts of 
never-relevant irrelevant information. These results are clearly consistent with the major arguments of the present report and, at the same time, are in sharp contrast to those obtained in the earlier studies of never-relevant irrelevant information. Morgan and Alluisi are apparently the first investigators to show that moderate amounts of such irrelevant information will degrade task performance. Their study seems to be the clearest available demonstration of the argument that competing responses are produced mainly by difficult discriminations and that the role of irrelevant information is primarily to enhance or amplify the competition. Evidence for this conclusion is provided by the finding that performance of their 0-irrelevant group declined systematically with increasing stimulus similarity. This condition permits the observation of response competition independent of any confounding between stimulus similarity and irrelevant information. The early studies of never-relevant irrelevant information were also free of this confounding, but unfortunately they used low-similarity stimuli in all irrelevancy conditions. The confounding is clearly present in the sometimes-relevant irrelevant experiments, but fortunately the significant interaction between the variables can be interpreted in a meaningful way.

In the Well (1971) investigation (Experiment I), significant performance decrements were produced by the number of irrelevant dimensions, the level of discrimination difficulty. and the dimension designated as relevant (three dimensions were used). Each of the double interactions of these variables was also statistically significant. Although the experiment was quite complicated, the results seem consistent with the present view that response competition is primarily the result of difficult discriminations. Inspection of Figs. 2 and 3 in Well suggest that the largest performance decrements are a function of the level of discriminability, even in the conditions with no irrelevant information. The figures also indicate that the irrelevant dimensions, irrespective of their prior relevance, had little effect on task performance, even though they accounted for significant variance in the statistical analysis. Furthermore, the lack of difference between Conditions 1 and 2 (changing vs constant relevant dimensions) reinforces the argument that irrelevant stimuli do not themselves engender response competition, but only amplify interference effects already present. Well's results, like those of Morgan and Alluisi, lend strong support to the critical role of difficult discriminations and attentuate the importance of irrelevant stimuli in the production of response competition.

\section{CONCLUSIONS}

The theoretical arguments of the present article represent an effort to provide a single explanatory framework for a growing set of seemingly contradictory findings about the effects of irrelevant information on identification and discrimination tasks. The proposed extension of the CR hypothesis in combination with the notion of information processing load seems reasonably consistent with the results of previous studies, but new investigations are needed to provide critical tests of the assertions. An independent demonstration, with new Ss and new stimulus materials, that systematic variation of stimulus similarity produces predictable changes in interference effects which can be increased or decreased by varying the amount of never-relevant irrelevant information would constitute strong support of the major assumptions of the present analysis. Given such a baseline, it would then be appropriate to examine more complex situations, e.g., tasks involving sometimes-relevant irrelevant information.

\section{REFERENCES}

Archer, E. J. Identification of visual patterns as a function of information load. Journal of Experimental Psychology, 1954. 48, 313-317.

Broadbent, D. E. Perception and communication. New York: Pergamon. 1958.

Egeth, H. E. Parallel versus serial processes in multidimensional stimulus discrimination. Perception \& Psychophysics, 1966. 1. 245-252.

Egeth. H. Selective attention. Psychological Bulletin, 1967.67. 41-57.

Egeth. H., \& Bevan, W. Attention. In B. Wolman (Ed.), Handbook of psychology. Englewood Cliffs, N.J: Prentice-Hall. 1972.

Ellis. H. The transfer of learning. New York: Macmillan. 1965.

Fitts. P. M. . \& Biederman. I. S-R compatibility and information reduction. Journal of Experimental Psychology, 1965, 69. 408-412.

Goldstein. I. Effects of stimulus complexity and restrictive responses. Journal of Experimental Psychology, 1966, 71, $104 \cdot 108$

Hodge, M. H. The influence of irrelevant information upon complex visual discrimination. Journal of Experimental Psychology, 1959, 57. 1-5.

Hodge. M. H.. \& Reid, L. S. The influence of similarity between relevant and irrelevant information upon a complex identification task. Perception \& Psychophysics. 1971, 10. 193-196.

Imai. S., \& Garner, W, R. Discriminability and preference for attributes in free and constrained classification. Joumal of Experimental Psychology, 1965, 69, 596-608.

MaGeoch. J. A.. \& Irion, A. L. The psychology of human learning. (2nd ed.) New York: Longmans, Green, 1952.

Montague, W. E. Effect of irrelevant information on a complex auditory-discrimination task. Journal of Experimental Psychology. 1965, 69. 230-236.

Morgan. B. B., Jr.. \& Alluisi. E. A. Effects of discriminability and irrelevant information on absolute judgments. Perception \& Psychophysics, 1967. 2. 54-58.

Morin, R. E.. Forrin, B., \& Archer, W. Information processing behavior: The role of irrelevant stimulus information. Journal of Experimental Psychology. 1961.61, 89-96.

Noble. C. E. Human trial-and-error learning. Psychological Reports. 1957. 3. 377-398.

Osgood. C. E. The similarity paradox in human learning: A resolution. Psychological Review. 1949. 56. 132-143.

Posner. M. I. Information reduction in the analysis of sequential tasks. Psychological Review. 1964. 71. 491-504. 
Posner. M. I., \& Boies, S. J. Components of attention. Psychological Review, 1971, 78, 391-408.

Postman. L. The present status of interference theory. In C. N. Cofer (Ed.). Verbal learning and verbal behavior. New York: McGraw-Hill. 1961. Pp. 152-179.

Stroop, J. R. Studies of interference in serial verbal reactions. Journal of Experimental Psychology, 1935, 18, 643-661.
Well, A. D. The influence of irrelevant information on speeded classification tasks. Perception \& Psychophysics, 1971, 10. 79-84.

(Received for publication November 13, 1972; accepted November 23, 1972.) 